\title{
Разработка Нечеткого OLAP-Куба в Хранилище Данных Системы Поддержки Принятия Решений
}

\author{
Гюльнара Набибекова \\ Институт Информационных Технологий НАНА \\ gulnarara58@mail.ru
}

\begin{abstract}
Аннотация- В статье показана интеграция технологии OLAP (Online Analytical Processing) и нечеткой логики при разработке нечеткого OLAP-куба В поликубической OLAР-модели в системе поддержки принятия решений. С этой целью фаззифицированы измерения OLAP-куба. Показано формирование нечеткого среза в результате нечеткого запроса. Также представлено практическое применение данного подхода в системе поддержки принятия решений при решении задачи управления кадровыми ресурсами.
\end{abstract}

Ключевые слова- система поддержки принятия решений, хранилище данных, OLAP, OLAP-кубЮ, нечеткое множество, функция принадлежности, терм-множсество, лингвистическая переменная, нечеткий запрос, нечеткий срез, индекс соответствия срезу

\section{I. ВВЕДЕНИЕ}

В процессе принятия решений можно столкнуться с множеством нечетких задач, вследствие чего в запросах к Хранилищам данных (ХД), которые формулирует аналитик, часто имеют место неточности и неопределенности. Кроме того, для лиц, принимающих решения, важен и полезен бывает не сам результат запроса, а то, является ли этот результат хорошим, средним или плохим, то есть им важна качественная сторона результата $[1,2]$. Все это требует, чтобы система поддержки принятия решений (СППР) могла использовать нечеткие и неопределенные рассуждения и поддерживала соответствующую технологию представления знаний [2]. В этом случае ХД должно обеспечивать ответы на запросы, которые использую лингвистические термины, являющиеся терммножествами соответствующей лингвистической переменной.

Для решения данной задачи необходимо выполнить фаззификацию измерений OLAP-куба и затем в результате нечеткого запроса сформировать нечеткий срез.

\section{II. ФАЗЗИФИКАЦИЯ ИЗМЕРЕНИЙ OLAР- КУБА}

В настоящее время для комплексного многомерного анализа больших объемов накопленной в ХД информации активно применяется технология OLAP [3]. Технология OLAP основана на представлении данных ХД в виде многомерной модели - гиперкуба, или OLAP-куба, содержащего одно или более измерений. Для фаззификации измерений OLAP-куба обратимся к получившему в настоящее время широкое распространение аппарату теории нечетких множеств в задачах поиска информации.

Нечеткое множество, включая функцию принадлежности, являющуюся его характеристикой, может быть задано экспертом. Но в некоторых случаях функцию принадлежности удобнее задать аналитической формулой и графически. Для задания функций принадлежности существуют различные типовые формы кривых, среди которых выберем треугольную и трапецеидальную кусочно-линейные формы. В общем случае треугольная функция принадлежности определяется тремя числами, а трапецеидальная - четырьмя, хотя их количество может и меняться в зависимости от условий задачи.

Для определения чисел, с помощью которых будет задана функция принадлежности, применим кластеризацию, на основании подхода, представленного в [1], согласно которому центры кластеров - медоиды, полученные в результате кластеризации соответствующих измерений, служат для определения функции принадлежности.

С целью выбора эффективного алгоритма кластеризации были рассмотрены неирархические алгоритмы, основанные на методе разбиения, а именно на методе k-medoids, так как медоид - это центр кластера, который принадлежит данному кластеру, что является главным условием задачи. Кроме того, методы k-medoids являются устойчивыми к наличию выбросов (outliers), т.е, точек, которые достаточно далеки от остальных точек, и могут работать достаточно эффективно с большими наборами данных.

Алгоритмы, основанные на методе разбиений, проходят два основных этапа [4]:

- начальный шаг, на котором k объектов выбраны в качестве медоидов;

- оценочный шаг, на котором происходит попытка минимизировать целевую функцию, обычно основанную на сумме общего расстояния между невыбранными объектами (значениями) и их медоидами, т.е.: 


$$
D(r, s)=\sum_{j=1}^{n} d\left(r_{i}, s_{j}\right)
$$

где $s_{j} \in S$ ( $S$ - множество объектов (значений) для кластеризации) и $d\left(r_{i}, s_{i}\right)<d\left(r_{c}, s_{j}\right), \forall r_{i}, r_{c} \in R(R-$ множество объектов (значений) из $\mathrm{S}$, выбранных в качестве медоидов), $r_{i} \neq r_{c}$. Чем меньше сумма расстояний между медоидом и всеми другими объектами соответствующего ему кластера, тем лучше кластеризация.

Были рассмотрены три известных алгоритма, основанных на методе k-medoids: PAM (Partitioning Around Medoids), CLARA (Clustering LARge Applications) и CLARANS (Clustering Large Applications based on RANdomized Search) [5, 6].

РАМ является одним из первых алгоритмов, основанных на методе k-medoids. В работах $[7,8]$ представлен основной принцип процесса кластеризации PАМ. заключающийся в переборе всех объектов, не являющихся медоидами, для вычисления расстояния от них до выбранных медоидов. Действие алгоритма РАМ приводит к высокому качеству кластеров, но поскольку он пробует все возможные комбинации, он эффективен для небольших наборов данных. И в связи с его вычислительной сложностью его применение непрактично для кластеризации больших наборов данных.

Вычислительная сложность алгоритма РАМ явилась мотивацией для разработки алгоритма CLARA алгоритма кластеризации на основе выборки [7]. CLARA выделяет из множества данных несколько выборок данных, применяет РАМ на каждой выборке и находит ее медоиды. Поскольку выборка произведена случайным образом, то медоиды выборки можно считать медоидами всего набора данных. Чтобы выбрать лучшие приближения, CLARA создает несколько выборок и на выходе выдает лучшую кластеризацию. В работе [7] также экспериментально показано, что пять выборок размером $(40+2 k)$, где $k-$ количество кластеров, дают удовлетворительные результаты.

CLARANS был разработан в рамках пространственного анализа данных. При поиске лучшего медоида на шаге оценки CLARANS случайным образом выбирает объекты из $(n-k)$ объектов $(n-$ количество объектов в множестве, $k$ - заданное количество кластеров, или количество медоидов). Количество объектов, перебираемых на этом этапе, ограничены параметром maxneighbor, заданным пользователем. Если после maxneighbor попыток не будет найдено лучшее решение, то локальный оптимум считается достигнутым. Процедура продолжается до тех пор, пока не найдено numloc локальных оптимумов.

Алгоритм CLARANS использует стратегию рандомизированного (случайного) поиска для облегчения кластеризации большого количества данных. Наличие большого количества данных позволяет гарантированно достичь numloc локальных оптимумов [8].

В результате исследования вышеупомянутых алгоритмов на предмет их достоинств и недостатков, для получения чисел, с помощью которых затем будет выполнена фаззификация, был выбран алгоритм CLARA, являющийся наиболее приемлемым для использования в данной задаче, с точки зрения сложности вычислений, размера множеств и затраченного времени.

Предположим, А является множеством измерений из всех измерений которые надо фаззифицировать.

Для каждого $j \epsilon A$ применим алгоритм CLARA и найдем в нем $k$ медоидов $a_{I j}, a_{2 j}, \ldots a_{k j}$ для $\mathrm{k}$ кластеров.

Пусть $J$ - множество значений $j$-го измерения;

$\mathrm{F}_{\mathrm{ij}}(\mathrm{x})$ - функция принадлежности для получения значений принадлежности всех значений $j$-го измерения в $i$-ом нечетком множестве. Рассмотрим случай, когда количество терм-множеств равно 3 , т.е. $i=3$. Отметим, что второе терм-множество считается средним, т.е. расположенным между первым и последним терммножествами.

Для всех $x \in \mathrm{J}$ выполняется:

1) для первого нечеткого множества (т.е. при $i=1$ )

$$
\begin{aligned}
& F_{l j}(x)=1, \text { если } x \leq a_{1 j} ; \\
& F_{l j}(x)=\left(a_{2 j}-x\right) /\left(a_{2 j}-a_{l j}\right), \text { если } a_{l j}<x<a_{2 j} ; \\
& F_{l j}(x)=0, \text { если } x \geq a_{2 j} .
\end{aligned}
$$

2) для второго нечеткого множества (т.е. при $i=2$ ) $F_{2 j}(x)=0$ если $x \leq a_{1 j}$;

$F_{2 j}(x)=\left(x-a_{1 j}\right) /\left(a_{2 j}-a_{1 j}\right)$, если $a_{1 j}<x<a_{2 j} ;$

$F_{2 j}(x)=1$, если $x=a_{2 j}$;

$F_{2 j}(x)=\left(a_{3 j}-x\right) /\left(a_{3 j}-a_{2 j}\right)$, если $a_{2 j}<x<a_{3 j} ;$

$F_{2 j}(x)=0$, если $x \geq a_{3 j}$.

3) для третьего нечеткого множества (т.е. при $i=3$ ) $F_{3 j}(x)=0$, если $x \leq a_{2 j}$;

$$
F_{3 j}(x)=\left(x-a_{2 j}\right) /\left(a_{3 j}-a_{2 j}\right) \text {, если } a_{2 j}<x<a_{3 j} ;
$$$$
F_{3 j}(x)=1 \text {, если } x \geq a_{3 j} \text {. }
$$

\section{III. ФОРМИРОВАНИЕ НЕЧЕТКОГО СРЕЗА}

В результате нечеткого запроса формируется нечеткий срез, состоящий из ячеек куба, соответствующих условиям запроса. Но для формирования итогового среза учитывают также и так называемый индекс соответствия срезу CI (Compliance Index), где $C I \in[0,1]$, который определяет аналитик [9].

Пусть имеется запрос: 
$\left\{\left(L_{1}=L_{1 j},\left(j=\overline{1, k_{1}}\right) \bigcup \bigcup\left(L_{2}=L_{2 j},\left(j=\overline{1, k_{2}}\right) \bigcup \bigcup \ldots \bigcup\left(L_{n}=L_{n j},\left(j=\overline{1, k_{n}}\right)\right)\right\}\right.\right.$, где $L_{1}, L_{2}, \ldots, L_{n}$ - лингвистические переменные, а $L_{1 j}$, $\left(j=\overline{\left.1, k_{1}\right)}, L_{2 j},\left(j=\overline{1, k_{2}}\right), \ldots, L_{n j},\left(j=\overline{1, k_{n}}\right)-\right.$ соответствующие им терм-множества.

В каждой записи ХД, $\forall x \in L_{i}$, где $i=\overline{1, n}$, то есть для любого $\mathrm{x}$ лингвистической переменной, участвующей в запросе, найдем степень принадлежности $\mu_{L_{i j}}(x)$ этого $x$ терм-множеству $L_{i j}$, участвующему в запросе.

Находим степень принадлежности каждой записи итоговому срезу, то есть ее индекс соответствия срезу, по формуле:

$$
C I=\min \left(\mu_{L_{m n}}(x)\right) .
$$

Для $C I$ вводятся условия, например:

если $C I \leq 2$, то соответствие срезу «слабое»;

если $2<C I<5$, то соответствие срезу «среднее»; если $C I \geq 5$, то принадлежность срезу «высокая».

\section{IV. ПРАКТИЧЕСКОЕ ПРИМЕНЕНИЕ}

В качестве примера создания нечеткого OLAP куба в поликубической OLAP-модели рассматривается подзадача управления кадровыми ресурсами в СППР, разработанной с целью определения степени интеграции стран. Данная СППР является многоцелевой, то есть помимо решения основной задачи, она может решать и побочные, в том числе и подзадачу управления кадровыми ресурсами, включающую решение таких вопросов, как анализ кадровых ресурсов, их размещение, обучение, планирование продвижения сотрудников и т.д. При решении такой подзадачи для лиц, принимающих решение, при формировании запроса к ХД часто бывает важен не конкретно возраст сотрудника, а то, является он молодымм, средним или пожильм, или, например, не конкретно стаж работы, а является он малым, средним или продолжительным. Кроме того, его может интересовать не точная дата мероприятия, в котором сотрудник принимал участие, а то, когда произошло это событие - давно или недавно.

Пусть с целью создания нечеткого OLAP-куба на основании соответствующей витрины данных из всего множества измерений надо фаззифицировать 3 измерения - ВОЗРАСТ, СТАЖ РАБОТЫ и ДАТА

В результате фаззификации измерения СТАЖ РАБОТЫ и ВОЗРАСТ становятся лингвистическими переменными, а полученные 3 кластера станут тремя терм-множествами. Для измерения СТАЖ РАБОТЫ это \{малый, средний, продолжительный\}, а для измерения ВОЗРАСТ это \{молодой, средний, пожилой

На рис.1 и рис.2 дано графическое изображение терм-множеств соответствующих измерений. Отметим, что номер $j$ следует закреплять за соответствующим измерением. В данном случае $j=1$ - измерение "ВОЗРАСТ", $j=2$ - измерение "СТАЖ РАБОТЫ".

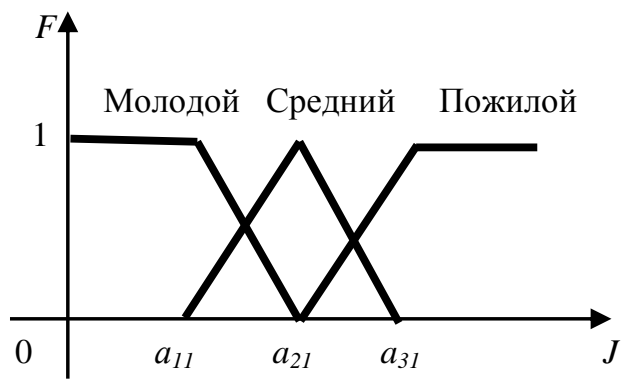

Рис. 1. Графическое изображение лингвистической переменной "BO3РАCT

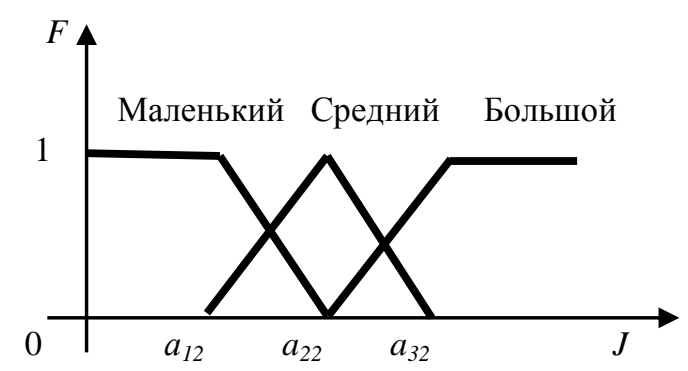

Рис. 2. Графическое изображение лингвистической переменной “СТАЖ РАБОТЫ”

Измерение ДАТА будет разбит на 2 терммножества (недавно $u$ давно), поэтому для его фаззификации применим алгоритм CLARA, задав количество кластеров $\mathrm{k}=2$. В результате будут получены 2 кластера с центрами (медоидами) в точках $\mathrm{a}_{13}$ и $\mathrm{a}_{23}, \mathrm{c}$ помощью которых будет задана функция принадлежности.

После выполнения фаззификации будут получены 2 терм-множества. На рис. 3 показано графическое изображение лингвистической переменной “ДАТА”.

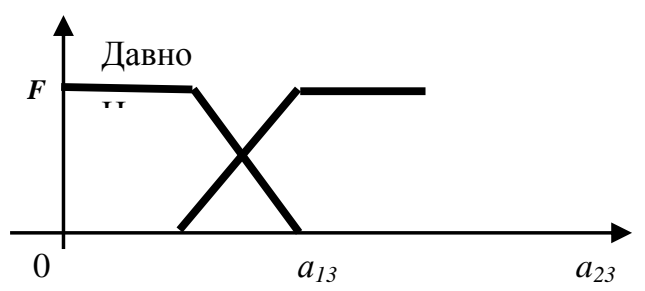

Рис. 3. Графическое изображение лингвистической переменной “ДАТА”.

В результате фаззификации измерений куба рассматриваемой СППР можно выполнять нечеткие запросы, используя термины молодой, зрельй, пожилой (касательно возраста), мальй, средний, продолжительный (касательно стажа работы), недавно и давно (касательно даты мероприятия). 
Приведены примеры типовых нечетких запросов, которые могут сыграть не последнюю роль в управления кадровыми ресурсами:

1. Молодые сотрудники, посетившие недавно Турцию;

2. Сотрудники, имеющие продолжительньй стаж работы, которые посетили недавно Турцию в связи с мероприятиями в медицинской сфере;

3. Страны, где молодые сотрудники прошли недавно тренинги в военной сфере и т.д.

\section{ЗАКЛЮЧЕНИЕ}

Данная СППР реализована для персональных компьютеров, работающих в среде Windows XP, Windows 7, Windows 8 и т.д., допускающей сетевое многопользовательское использование технологии клиент-сервер. В качестве платформы используется система управления базами данных MS SQL сервер [10]. Среда реализации OLAP - Microsoft Visual Studio 2008 (Analysis Services). Клиентская часть реализована на языке программирования Object Pascal [11] с применением среды разработки приложений Delphi 2010 [12] и предназначена для обращения к серверу, обработки и представления полученных данных. Используемый язык запросов - T-SQL [13].

\section{ЛИТЕРАТУРА}

[1] K. Kumar et al. Fuzzy OLAP Cube for Qualitative Analysis / 3rd International Conference on Intelligent Sensing and Information Processing (ICISIP), 2005, p. $290 \quad-\quad 295$. http://ieeexplore.ieee.org/xpls/abs_all.jsp?arnumber=1529464\&tag=1

[2] Sh. Wang. Application of Decision Support System in E-government / International Conference "Management and Service Science", 2009, (MASS'09) http://ieeexplore.ieee.org/stamp/stamp.jsp?tp=\&arnumber $=5302532$

[3] E. F. Codd, S. B. Codd, C.T. Salley. Providing OLAP (Online Analytical Processing) to User-Analysts: An IT Mandate. San Jose: Codd \& Date, Inc., 1993, p. 31. www.minet.unijena.de/dbis/lehre/ss2005/sem_dwh/lit/Cod93.pdf
[4] M.-C. N. Barioni et al. An efficient approach to scale up k-medoid based algorithms in large databases. XXI Brazilian Simposium on Databases. www.lbd.dcc.ufmg.br:8080/colecoes/sbbd/2006/018.pdf

[5] L. Kaufman, P. J. Rousseeuw Finding Groups in Data: an Introduction to Cluster Analysis. John Wiley \& Sons, 2005,

[6] R.T. Ng, J. Han. Clarans: A method for clustering objects for spatial data mining // IEEE Transactions on Knowledge and Data Engineering (TKDE), 2002, 14(5), pp. 1003-1016.

[7] Г. Набибекова. Об одном методе фаззификации атрибутов хранилища данных в системах поддержки принятия решений в сфере внешней политики // Информационные технологии. №1, 2014, c.36-41.

[8] Г. Набибекова Об одном подходе к разработке нечеткого OLAPкуба в системах поддержки принятия решений в сфере внешней политики / Материалы IV Международной научно-практической конференции “Проблемы кибернетики и информатики” РСI2012, 2012, c.86-89.

[9] Н. Б. Паклин, В. И. Орешков. Бизнес-аналитика: от данных к знаниям. Учебное пособие, Питер, 2013, 703 с.

[10] R. Dyer Learning MySQL and MariaDB: Heading in the Right Direction with MySQL and MariaDB. O'Reilly Media, 2015, 408 p.

[11] M. Cantu Object Pascal Handbook. CreateSpace Independent Publishing Platform, 2015, $552 \mathrm{p}$.

[12] N. Hodges. More Coding in Delphi. Nepeta Enterprises, 2015, 246 p.

[13] I. Ben-Gan. T-SQL Fundamentals. Microsoft Press, 2016, 464 p. 